\title{
Membranous Nephropathy: It Is Time to Go Back to the Future
}

\author{
Gianmarco Sabiu ${ }^{\mathrm{a}}$ Manuel Alfredo Podestà ${ }^{b}$ \\ aNephrology Unit, ASST Fatebenefratelli Sacco, Department of Biomedical and Clinical Sciences "Luigi Sacco,"

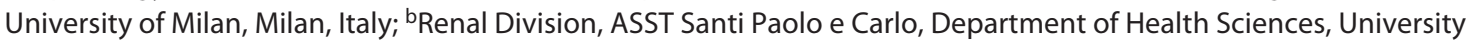 \\ of Milan, Milan, Italy
}

A comment on "Scolari et al.: Rituximab or Cyclophosphamide in the Treatment of Membranous

Nephropathy: The RI-CYCLO Randomized Trial. J Am Soc Nephrol. 2021 Mar;32(4):972-82.”

\section{Keywords}

Membranous nephropathy · Rituximab ·

Cyclophosphamide $\cdot$ Corticosteroids · Therapy

\begin{abstract}
Context: Membranous nephropathy (MN) is an immune-mediated glomerular disease that can lead to nephrotic syndrome and progressive kidney function loss. The cyclic steroid-cyclophosphamide regimen (the modified Ponticelli protocol) and the monoclonal anti-CD20 antibody rituximab have been advocated as effective therapies to improve renal outcomes, but a direct comparison of these treatments had never been carried out in a prospective study. Subject of $R \boldsymbol{e}-$ view: Scolari et al. [J Am Soc Nephrol. 2021;32:972-82] recently reported the results of a pilot randomized controlled trial (RI-CYCLO) designed to provide direct estimates of the effect of rituximab $(1 \mathrm{~g} \times 2$ ) compared to the cyclic steroid-cyclophosphamide regimen in 74 patients with $\mathrm{MN}$. The proportion of patients with complete remission at 12 months was higher in the cyclic regimen arm than that of rituximab (32 and $16 \%$, respectively), but the difference was not statistically significant in intention-to-treat analyses. Interestingly, differences in the cumulative incidence of complete and partial remissions between treatment arms progressively re-
\end{abstract}

duced over the follow-up and became virtually nonexistent from 24 months (>80\% in both groups). The frequency of serious and nonserious adverse events was similar between the 2 treatment arms. Infusion reactions and drug discontinuation were more common with rituximab, while infections and leukopenia were more frequently observed with the cyclic regimen. The risk of cancer was similar in the $2 \mathrm{al}-$ location groups, but the limited follow-up length did not allow to draw definitive conclusions. Independent of treatment allocation, $18 \%$ of patients experienced at least 1 relapse after achieving complete or partial remission. Second Opinion: Notwithstanding the intrinsic limitations of a pilot study, the RI-CYCLO trial represents an important milestone in the treatment of MN. Findings from this study support the hypothesis that the cyclic regimen and rituximab may have comparable efficacy in inducing disease remission over the long term. Considering its potentially better-albeit not yet formally proven-long-term safety profile, rituximab could be considered as a first-line therapy for most patients with MN. Several questions remain to be addressed, including rituximab ideal dose and its efficacy in patients with a significant reduction in glomerular filtration rate. In light of RICYCLO results, a large-scale trial to assess rituximab noninferiority to the cyclic regimen would require the enrollment of thousands of patients, and it would be probably unfeasi- 
ble within a reasonable time frame. In our opinion, resources should be allocated to provide an answer to the pressing matter of treatment nonresponse and intolerance, which may be addressed in the near future with novel therapeutic strategies.

(c) 2021 S. Karger AG, Basel

\section{Autoimmunity in Membranous Nephropathy}

Membranous nephropathy ( $\mathrm{MN})$ is the leading cause of nephrotic syndrome in adults, affecting 1 patient per 100,000 population. $\mathrm{MN}$ is an immune-mediated glomerular disease characterized by subepithelial deposition of podocyte-targeted antibodies, which induce a progressive damage to the glomerular capillary wall, leading to proteinuria and kidney failure. Almost $30 \%$ of MN diagnoses are associated with malignancy, systemic autoimmune diseases, infections, or drugs, while the remaining cases have been traditionally defined as primary forms [1].

Despite its defining histological features, $\mathrm{MN}$ is a heterogeneous autoimmune disorder. Several podocyte antigens have been recognized as specific autoantibody targets, including phospholipase $\mathrm{A}_{2}$ receptor $\left(\mathrm{PLA}_{2} \mathrm{R}\right)$, thrombospondin type 1 domain-containing 7A, and neural epidermal growth factor like-1 protein, which are detectable in $75-85 \%$ of primary MN cases. More recently, exostosin 1 /exostosin 2 , protocadherin 7 , neural cell adhesion molecule 1 , and semaphorin-3B have been also identified as potential MN target proteins/antigens. Since each of these is reportedly associated with distinct clinical features and outcomes, MN may represent a pattern of injury common to several different pathogenetic mechanisms rather than a single disease [2].

The natural history of $\mathrm{MN}$ is highly variable. Although $30 \%$ of patients may experience spontaneous disease remission, $\mathrm{MN}$-associated nephrotic syndrome can severely impact on renal survival, with one-third of patients progressing to end-stage kidney disease within 10 years from diagnosis [3, 4]. Immunosuppressive treatment should be reserved to patients at high risk of progressive kidney injury, but the best treatment approach remains controversial due to difficulties in discriminating between progressive and nonprogressive disease.

Alkylating agents (cyclophosphamide or chlorambucil) combined with steroids were the first immunosuppressive drugs shown to increase the remission rate of $\mathrm{MN}$-associated nephrotic syndrome compared to supportive treatment or steroids alone [5-8]. These agents can prevent kidney function loss in patients with progres- sive disease [9], and the currently recommended cyclic corticosteroid-cyclophosphamide regimen may increase long-term dialysis-free survival in MN patients (Table 1) [4]. However, the use of alkylating agents may be associated with a higher risk of infection, cancer, and infertility due to the toxic effect of their metabolites.

Advances in the understanding of MN pathophysiology suggested that direct targeting of B cells could be exploited to inhibit the production of nephritogenic autoantibodies. After the first successful reports [10], cohort studies showed that rituximab, a B-cell-depleting chimeric anti-CD20 monoclonal antibody, could induce nephrotic syndrome remission in a significant fraction of $\mathrm{MN}$ patients [11]. In recent years, 3 randomized controlled trials (RCTs) assessed rituximab efficacy and safety in MN (Table 2). The GEMRITUX and MENTOR trials reported the superiority of rituximab in inducing and maintaining nephrotic syndrome remission compared to either supportive therapy alone or cyclosporine $[12,13]$. In the STARMEN trial, the cyclic corticosteroid-cyclophosphamide regimen was more effective in inducing MN remission than a sequential therapy with tacrolimus and rituximab [14]. However, the STARMEN design entailed a single rituximab dose that was infused at 6 months to prevent relapses after tacrolimus weaning, thus not allowing to reach a definitive conclusion on the relative efficacy of rituximab compared to the cyclic regimen. Up to this point, a head-to-head direct comparison of these 2 treatment strategies was lacking.

\section{Key Answers and Open Questions from the RI-CYCLO Trial}

The RI-CYCLO study was designed to provide, for the first time in the RCT setting, direct estimates of the effect of rituximab compared to the cyclic regimen in $\mathrm{MN}$ patients [15]. The trial recruited immunosuppression-naive adult subjects with biopsy-proven $\mathrm{MN}$ and nephroticrange proteinuria $(>3.5 \mathrm{~g} / 24 \mathrm{~h}$ ). Patients with diabetes or severely reduced renal function were excluded from the study. After a run-in period of 3 months, 74 participants were randomized 1:1 to receive either 2 infusions of rituximab ( $1 \mathrm{~g}, 2$ weeks apart) or the cyclic regimen (corticosteroids on months 1,3 , and 5 and oral cyclophosphamide $2.0 \mathrm{mg} / \mathrm{kg} /$ day on months 2 , 4 , and 6). The primary outcome of the trial was the incidence of complete remission at 12 months, but the follow-up was extended to 36 months to capture delayed treatment responses. Owing to dropouts and to the long screening period (84 months), 

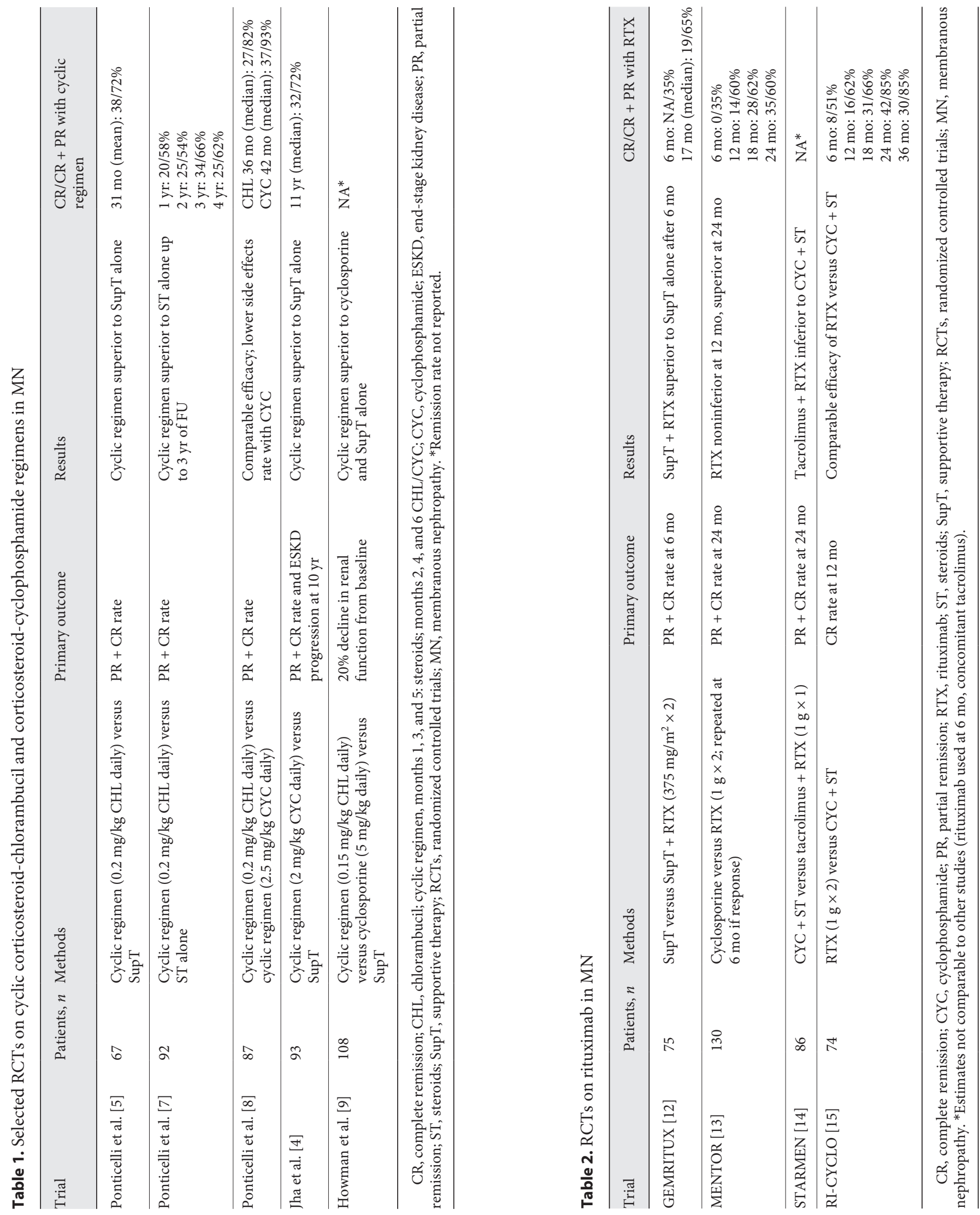
which underscore the hurdles of conducting academic multicenter trials on rare diseases, only 42 patients had complete data for the whole follow-up period.

The proportion of patients with complete remission at 12 months was higher in the cyclic regimen arm than in rituximab (32 and 16\%, respectively), but the difference was not statistically significant at intention-to-treat analyses. Despite of a more rapid depletion of anti-PLA ${ }_{2} \mathrm{R}$ antibodies in the rituximab group, there was a trend toward faster remission in patients allocated to the cyclic regimen. By assessing the cumulative incidence of complete and partial remissions, the gap between the 2 treatment groups became progressively smaller during the followup and was virtually nonexistent at 24 months (0.82 and 0.83 , respectively), underscoring the importance of an adequate follow-up length in MN trials. Per protocol analyses reported a higher probability of complete remission at 12 months for patients allocated to the cyclic regimen, but this group also included 3 patients originally randomized to rituximab who did not complete the treatment due to adverse events and were switched to the cyclic regimen. In subgroup analyses, there was a trend toward a higher efficacy of the cyclic regimen in patients with more severe nephrotic syndrome. However, none of the interactions between treatment allocation and nephrotic syndrome features was statistically significant.

Within the limits of a relatively short pilot trial, this study also provides valuable insight into the safety of rituximab versus the cyclic regimen. Although the frequency of serious and nonserious adverse events was similar between the 2 treatment groups, the type of events differed. Infusion reactions to the drug were the most common adverse events in the rituximab group (24\% of participants), leading to permanent discontinuation in 4 (11\%) cases. The frequency of infusion reactions in the MENTOR trial was similar (25\%), but these led to discontinuation only in $2(3 \%)$ patients. On the other hand, the GEMRITUX did not report any hypersensitivity reaction to rituximab, and none of the participants discontinued the treatment, suggesting a certain degree of variability among patient cohorts.

The cyclic regimen was well-tolerated by all patients except one, who was intolerant to cyclophosphamide. Leukopenia and infections including pneumonia were more frequent in participants allocated to the cyclic regimen, but the incidence of adverse events was lower than the one previously reported in a large retrospective cohort study [16]. Notably, lower cumulative cyclophosphamide doses were used in the RI-CYCLO trial, and weekly monitoring of leukocyte counts followed by dose adjustments may have played a role in preventing further complications. Nonetheless, the risk of gonadal toxicity should still be considered when treating younger patients, as the general threshold for infertility risk $(10 \mathrm{~g})$ is usually reached even with this regimen [17]. Three patients developed cancer during the study, 2 in the rituximab and 1 in the cyclic regimen group, suggesting a similar risk profile. However, the follow-up length of this pilot trial does not allow to assess the long-term risk of malignancy. Long-term safety data on the low-dose cyclic steroid-cyclophosphamide regimen suggest that the risk of cancer at 10 years from treatment is very low, but these figures are derived from a single monocentric RCT in which treatment dates back to the early 1990s [4].

Several key questions remain to be addressed. The best rituximab dose for $\mathrm{MN}$ is still a matter of debate, with a single retrospective study suggesting that higher rituximab doses could lead to more rapid remission [18]. Despite lower rituximab doses in the RI-CYCLO compared to the MENTOR trial, complete and partial remission rates were similar between the 2 studies. However, subjects enrolled in the RI-CYCLO trial had a relatively more benign $\mathrm{MN}$ phenotype, that is, lower baseline proteinuria and anti-PLA ${ }_{2} \mathrm{R}$ levels, which may have acted as confounders. Results of the RI-CYCLO trial may not be generalizable to all patients: diabetic subjects were excluded from the study, and most patients enrolled had normal renal function. The risk of steroid side effects may be higher in diabetic patients, and alkylating agent toxicity could increase in patients with reduced renal function [9]. Nonetheless, safety and efficacy data on rituximab in these patients are also lacking.

Overall, rituximab and the cyclic regimen led to similar results in $\mathrm{MN}$ patients, and differences in their longterm potential to induce remission-if any-are likely minor. We agree with the authors that, considering its potentially better long-term safety profile, rituximab may be considered as a first-line therapy in most MN patients.

What about a follow-up noninferiority trial after this pilot study? Notably, by assuming a reasonable noninferiority margin, the authors calculated that an adequately powered trial would require the inclusion of thousands of participants to assess rituximab noninferiority to the cyclic regimen. Moreover, since the efficacy of the 2 regimens at 24 months in this pilot study was virtually identi$\mathrm{cal}$, this figure would probably need to be increased further in order to obtain meaningful results. Considering $\mathrm{MN}$ incidence and the issues connected to a multinational trial, we argue that such a study would be probably unfeasible in a reasonable time frame. In our opinion, re- 


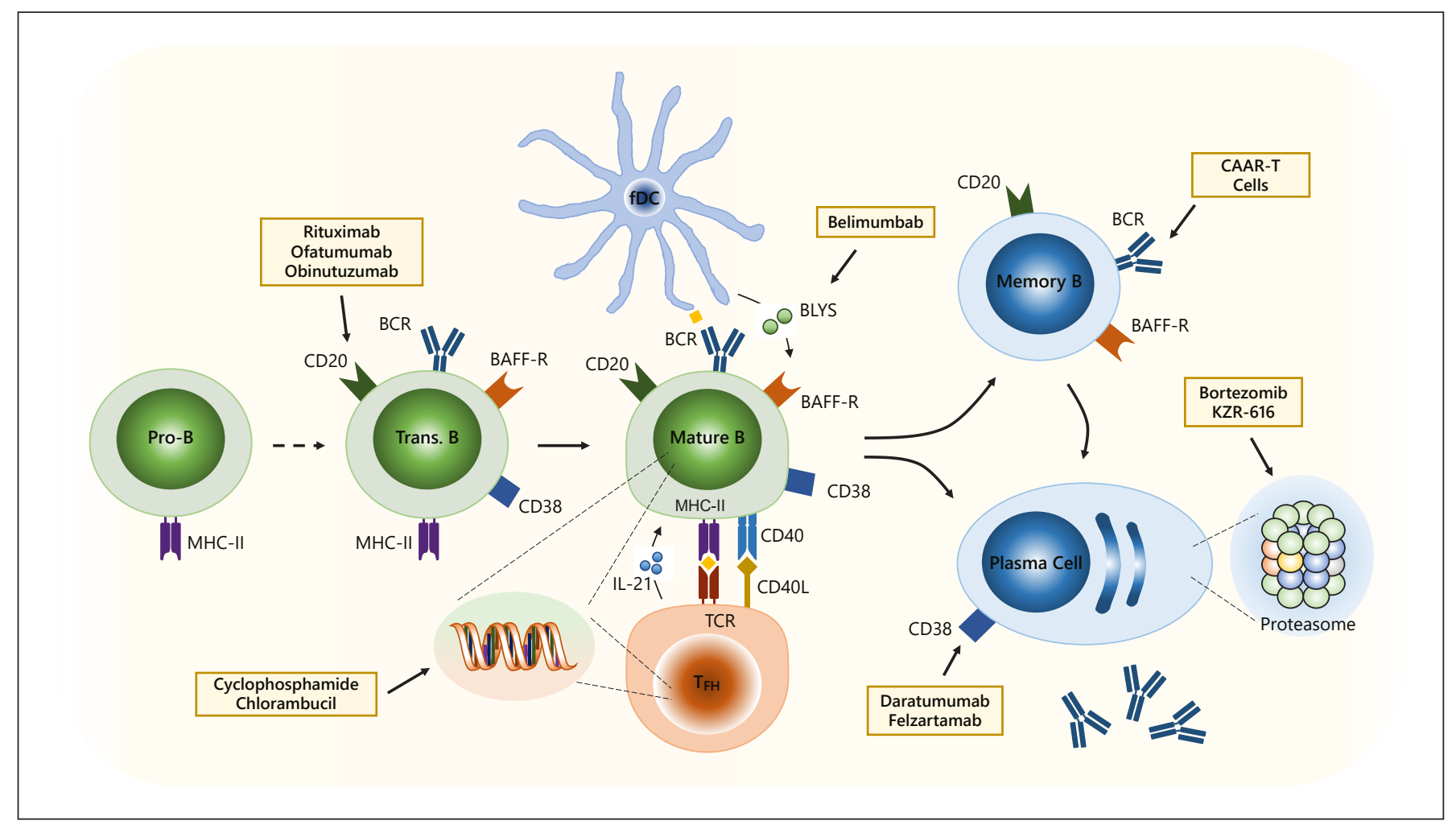

Fig. 1. Simplified representation of established and potential therapeutic targets in MN. Notably, corticosteroids have multiple genomic and nongenomic effects, which have not been depicted. BAFF-R, BAFF receptor; BCR, B-cell receptor; Blys, soluble B-lymphocyte stimulator; fDC, follicular dendritic cell; MHC-II, class II major histocompatibility complex; TCR, T-cell receptor; $\mathrm{T}_{\mathrm{FH}}$, follicular helper T cell; Trans. B, transitional B cell; $\mathrm{MN}$, membranous nephropathy.

sources should be allocated to provide an answer to the pressing matter of treatment nonresponse and intolerance.

\section{A Glimpse into the Future}

Despite the efficacy of current therapies, $20-40 \%$ of $\mathrm{MN}$ patients may not respond to rituximab or the cyclic regimen. In addition, disease relapses are frequent $(18 \%$ during the RI-CYCLO trial follow-up), and repeated treatment can be complicated by hypersensitivity reactions elicited by rituximab murine component or systemic toxicity due to cyclophosphamide and steroid off-target effects.

Treatment resistance and disease relapses may be explained by incomplete or insufficient depletion of autoreactive B-cell clones in lymphoid organs, leading to ongoing differentiation into plasma cells with persistent or re- current production of nephritogenic antibodies (Fig. 1). Consistent with this hypothesis, several studies have shown that despite complete peripheral B-cell depletion, rituximab rarely achieves the same effect in lymphoid tissues $[19,20]$.

Novel monoclonal antibodies have been designed to increase specific lytic activity against cells bearing the CD20 molecule. These drugs have been also humanized to overcome the risk of immune responses against xenogeneic antibody components. Ofatumumab is a fully human anti-CD20 antibody that exerts greater complement-dependent cytotoxicity than rituximab. Anecdotal reports have shown that this agent could induce remission both in $\mathrm{MN}$ patients intolerant to rituximab and in those who became resistant to the drug due the emergence of anti-chimeric antibodies [21,22].

Obinutuzumab is a type II anti-CD20 glycoengineered antibody that depletes B cells through antibody-dependent cell-mediated cytotoxicity [23]. Owing to its en- 
hanced B-cell-depleting potential in lymphoid organs, obinutuzumab could theoretically boost immunologic remission in rituximab nonresponders. Two small case series reported encouraging results in rituximab-resistant and rituximab-intolerant $\mathrm{MN}$ patients [24, 25]. A multicentric, randomized, open-label, phase-III trial comparing the efficacy of obinutuzumab to tacrolimus in $\mathrm{MN}$ subjects is underway (NCT04629248).

Aside from depletion, targeting B-cell differentiation and homeostasis could represent a complementary strategy to induce $\mathrm{MN}$ remission. Belimumab, a monoclonal antibody that antagonizes the biological activity of the soluble B-lymphocyte stimulator, proved effective as an add-on therapy for lupus nephritis [26]. A small prospective study in 14 patients with primary MN showed that belimumab monotherapy was associated with a reduction in anti-PLA ${ }_{2} \mathrm{R}$ levels, leading to remission in $64 \%$ of subjects at 2 years [27]. The REBOOT trial (NCT03949855) will investigate the efficacy of a combined therapy with belimumab and rituximab compared to rituximab alone for the treatment of primary MN.

Another possible explanation of treatment resistance in some MN patients might be the presence of autoreactive long-lived plasma cells, whose maintenance is at least partially independent of activated and memory B cells. Rituximab does not directly deplete plasma cells due to their lack of CD20 expression. Similarly, plasma cell resistance to alkylating agents can be relatively high due to their terminally differentiated state and increased expression of aldehyde dehydrogenase.

Targeting of plasma cells with bortezomib, a constitutive proteasome inhibitor that induces apoptosis in cells with a high-protein turnover, has been successful in selected cases of treatment-resistant MN [28]. However, the use of bortezomib has been discouraged by the high risk of adverse events. Off-target effects could be minimized by directly targeting the immunoproteasome, an alternative form of the proteasome that is constitutively expressed only in immune cells. After encouraging preclinical results, immunoproteasome inhibitors are currently being tested in lupus nephritis (NCT03393013) but might be also beneficial to $\mathrm{MN}$ patients in the future.

Since plasma cells are characterized by high CD38 expression, direct targeting of this molecule could prove effective in controlling MN autoimmunity. Daratumumab, an anti-CD38 antibody currently approved for the treatment of multiple myeloma, was successfully employed in a small case series of patients with lupus nephritis [29]. A recent phase II study using daratumumab for proliferative glomerulonephritis with monoclonal immunoglobu- lin deposits also reported a high efficacy [30]. Two trials exploring the effects of another anti-CD38 antibody, Felzartamab, in patients with $\mathrm{PLA}_{2} \mathrm{R}$-related $\mathrm{MN}$ are currently ongoing (NCT04145440, NCT04733040).

Finally, the discovery of antigen specificities has opened the frontier of antigen-specific treatment for MN. An attractive opportunity may derive from the development of chimeric autoantibody receptor T cells, which are engineered to express the autoantigen of interest fused to T-cell signaling domains [31]. Chimeric autoantibody receptor T cells target and lyse autoreactive B cells bearing an autoantigen-specific B-cell receptor, leaving the rest of the B-cell pool unaffected. This strategy could theoretically induce remission without the risks associated with generalized immunosuppression.

Albeit promising, it is unlikely that any of these therapies will be able to induce remission in all $\mathrm{MN}$ patients. As $\mathrm{MN}$ is being increasingly recognized as a spectrum of disorders, we will have to gradually abandon the onetherapy-fits-all concept. Biomarkers of response beyond serum antibody monitoring are urgently required, and future trials will need to focus on patient stratification to identify the most promising strategies in different $\mathrm{MN}$ subsets.

\section{Conflict of Interest Statement}

The authors have no conflict of interest to declare.

\section{Funding Sources}

No relevant funding sources to report.

\section{Author Contributions}

G.S. and M.A.P. jointly wrote the first draft of the manuscript and revised its content critically.

References

1 Ronco P, Debiec H. Molecular pathogenesis of membranous nephropathy. Annu Rev Pathol. 2020 Jan;15:287-313.

2 Sethi S. Membranous nephropathy: single disease or a pattern of injury resulting from different diseases. Clin Kidney J. 2021 Mar.

3 Schieppati A, Mosconi L, Perna A, Mecca G, Bertani T, Garattini S, et al. Prognosis of untreated patients with idiopathic membranous nephropathy. N Engl J Med. 1993 Jul;329(2): 85-9. 
4 Jha V, Ganguli A, Saha TK, Kohli HS, Sud K, Gupta KL, et al. A randomized, controlled trial of steroids and cyclophosphamide in adults with nephrotic syndrome caused by idiopathic membranous nephropathy. J Am Soc Nephrol. 2007 Jun; 18(6):1899-904.

5 Ponticelli C, Zucchelli P, Imbasciati E, Cagnoli L, Pozzi C, Passerini P, et al. Controlled trial of methylprednisolone and chlorambucil in idiopathic membranous nephropathy. $\mathrm{N}$ Engl J Med. 1984 Apr;310(15):946-50.

6 Ponticelli C, Zucchelli P, Passerini P, Cesana B, Locatelli F, Pasquali S, et al. A 10-year follow-up of a randomized study with methylprednisolone and chlorambucil in membranous nephropathy. Kidney Int. 1995 Nov; 48(5):1600-4.

7 Ponticelli C, Zucchelli P, Passerini P, Cesana B. Methylprednisolone plus chlorambucil as compared with methylprednisolone alone for the treatment of idiopathic membranous nephropathy. The Italian idiopathic membranous nephropathy treatment study group. N Engl J Med. 1992 Aug;327(9):599603.

8 Ponticelli C, Altieri P, Scolari F, Passerini P, Roccatello D, Cesana B, et al. A randomized study comparing methylprednisolone plus chlorambucil versus methylprednisolone plus cyclophosphamide in idiopathic membranous nephropathy. J Am Soc Nephrol. 1998 Mar;9(3):444-50.

9 Howman A, Chapman TL, Langdon MM, Ferguson C, Adu D, Feehally J, et al. Immunosuppression for progressive membranous nephropathy: a UK randomised controlled trial. Lancet. 2013 Mar;381(9868):744-51.

10 Remuzzi G, Chiurchiu C, Abbate M, Brusegan V, Bontempelli M, Ruggenenti P. Rituximab for idiopathic membranous nephropathy. Lancet. 2002 Sep;360(9337):923-4.

11 Ruggenenti P, Cravedi P, Chianca A, Perna A, Ruggiero B, Gaspari F, et al. Rituximab in idiopathic membranous nephropathy. J Am Soc Nephrol. 2012 Aug;23(8):1416-25.

12 Dahan K, Debiec H, Plaisier E, Cachanado M, Rousseau A, Wakselman L, et al. Rituximab for severe membranous nephropathy: a 6-month trial with extended follow-up. J Am Soc Nephrol. 2017 Jan;28(1):348-58.
13 Fervenza FC, Appel GB, Barbour SJ, Rovin $\mathrm{BH}$, Lafayette RA, Aslam N, et al. Rituximab or cyclosporine in the treatment of membranous nephropathy. N Engl J Med. 2019 Jul; 381(1):36-46.

14 Fernández-Juárez G, Rojas-Rivera J, Logt AV, Justino J, Sevillano A, Caravaca-Fontán F, et al. The STARMEN trial indicates that alternating treatment with corticosteroids and cyclophosphamide is superior to sequential treatment with tacrolimus and rituximab in primary membranous nephropathy. Kidney Int. 2021 Apr;99(4):986-98.

15 Scolari F, Delbarba E, Santoro D, Gesualdo L, Pani A, Dallera N, et al. Rituximab or cyclophosphamide in the treatment of membranous nephropathy: the RI-CYCLO randomized trial. J Am Soc Nephrol. 2021 Mar;32(4): 972-82.

16 van den Brand JAJG, Ruggenenti P, Chianca A, Hofstra JM, Perna A, Ruggiero B, et al. Safety of rituximab compared with steroids and cyclophosphamide for idiopathic membranous nephropathy. J Am Soc Nephrol. 2017 Sep;28(9):2729-37.

17 Ponticelli C, Escoli R, Moroni G. Does cyclophosphamide still play a role in glomerular diseases? Autoimmun Rev. 2018 Oct;17(10): $1022-7$.

18 Seitz-Polski B, Dahan K, Debiec H, Rousseau A, Andreani M, Zaghrini C, et al. High-dose rituximab and early remission in PLA2R1-related membranous nephropathy. Clin J Am Soc Nephrol. 2019 Aug;14(8):1173-82.

19 Kamburova EG, Koenen HJ, Borgman KJ, ten Berge IJ, Joosten I, Hilbrands LB. A single dose of rituximab does not deplete B cells in secondary lymphoid organs but alters phenotype and function. Am J Transplant. $2013 \mathrm{Jun}$; 13(6):1503-11.

20 Wallin EF, Jolly EC, Suchánek O, Bradley JA, Espéli M, Jayne DR, et al. Human T-follicular helper and T-follicular regulatory cell maintenance is independent of germinal centers. Blood. 2014 Oct;124(17):2666-74.

21 Podestà MA, Ruggiero B, Remuzzi G, Ruggenenti P. Ofatumumab for multirelapsing membranous nephropathy complicated by rituximab-induced serum-sickness. BMJ Case Rep. 2020 Jan;13(1):e232896.
22 Boyer-Suavet S, Andreani M, Lateb M, Savenkoff B, Brglez V, Benzaken S, et al. Neutralizing anti-rituximab antibodies and relapse in membranous nephropathy treated with rituximab. Front Immunol. 2019;10:3069.

23 Redfield RR, Jordan SC, Busque S, Vincenti F, Woodle ES, Desai N, et al. Safety, pharmacokinetics, and pharmacodynamic activity of obinutuzumab, a type 2 anti-CD20 monoclonal antibody for the desensitization of candidates for renal transplant. Am J Transplant. 2019;19(11):3035-45.

24 Klomjit N, Fervenza FC, Zand L. Successful treatment of patients with refractory PLA2Rassociated membranous nephropathy with obinutuzumab: a report of 3 cases. Am J Kidney Dis. 2020 Dec;76(6):883-8.

25 Sethi S, Kumar S, Lim K, Jordan SC. Obinutuzumab is effective for the treatment of refractory membranous nephropathy. Kidney Int Rep. 2020 Jul;5(9):1515-8.

26 Furie R, Rovin BH, Houssiau F, Malvar A, Teng YKO, Contreras G, et al. Two-year, randomized, controlled trial of belimumab in lupus nephritis. N Engl J Med. 2020 Sep; 383(12):1117-28.

27 Barrett C. Effect of belimumab on proteinuria and anti-phospholipase A2 receptor autoantibody in primary membranous nephropathy. Nephrol Dial Transplant. 2019.

28 Barbari A, Chehadi R, Kfoury Assouf H, Kamel G, Jaafar M, Abdallah A, et al. Bortezomib as a novel approach to early recurrent membranous glomerulonephritis after kidney transplant refractory to combined conventional rituximab therapy. Exp Clin Transplant. 2017 Jun;15(3):350-4.

29 Ostendorf L, Burns M, Durek P, Heinz GA, Heinrich F, Garantziotis P, et al. Targeting CD38 with daratumumab in refractory systemic lupus erythematosus. N Engl J Med. 2020 Sep;383(12):1149-55.

30 Zand L, Rajkumar SV, Leung N, Sethi S, El Ters M, Fervenza FC. Safety and efficacy of daratumumab in patients with proliferative GN with monoclonal immunoglobulin deposits. J Am Soc Nephrol. 2021 Mar;32(5): 1163-1173.

31 Ellebrecht CT, Bhoj VG, Nace A, Choi EJ, Mao X, Cho MJ, et al. Reengineering chimeric antigen receptor $\mathrm{T}$ cells for targeted therapy of autoimmune disease. Science. 2016 Jul; 353(6295):179-84. 